\title{
Elevated Cystatin-C Levels Are Associated with Increased Mortality in Acute Coronary Syndrome Patients: An HIJ-PROPER Sub-Analysis
}

\author{
Takashi Saito $^{a, b}$ Hiroyuki Arashi ${ }^{b}$ Junichi Yamaguchi ${ }^{b}$ Fumiaki Mori ${ }^{a}$ \\ Hiroshi Ogawa $^{\mathrm{b}}$ Nobuhisa Hagiwara ${ }^{\mathrm{b}}$ \\ ${ }^{a}$ Cardiology, National Hospital Organization Yokohama Medical Center, Yokohama, Japan; ${ }^{b}$ Department of \\ Cardiology, The Heart Institute of Japan, Tokyo Women's Medical University, Tokyo, Japan
}

\section{Keywords}

Cystatin-C · Mortality · Cardiovascular events · Acute coronary syndrome

\begin{abstract}
Background and Aims: We investigated the association between serum cystatin-C (Cys-C) levels and cardiovascular events in patients with acute coronary syndrome (ACS). Methods: Data of 1,100 patients from the prospective parent study were included. Patients hospitalized for ACS were divided into 4 groups based on quartiles (Q) of Cys-C levels (mg/L) within $24 \mathrm{~h}$ of admission: Q1, $\leq 0.82 ; \mathrm{Q} 2,0.82<$ estimated level $\leq 0.95 ;$ Q3, $0.95<$ estimated level $\leq 1.12$; and Q4, $>1.12$. The primary endpoint of this study was all-cause mortality, and the secondary endpoint was composite of allcause mortality, nonfatal myocardial infarction, nonfatal stroke, unstable angina pectoris, or ischemia-driven revascularization. Results: During a median observation period of 4.0 years, the primary endpoint was noted in $5,12,18$, and 36 patients in Q1-Q4, respectively, with corresponding incidence rates of $1.8 \%, 4.4 \%, 6.5 \%$, and $13.5 \%$, respectively $(p<$ 0.0001 for difference among 4 groups). This association persisted even after adjusting for patient characteristics and other laboratory results at baseline $(p=0.04)$. A stepwise increase in the incidence rate of the secondary endpoint with
\end{abstract}

an incline in Cys-C levels was observed in the nonadjusted model $(26.6 \%, 33.3 \%, 32.3 \%$, and $39.1 \%$ in Q1-Q4, respectively; $p=0.01$ ) but not in the adjusted model ( $p=0.3$ ). No difference was observed in the incidence rate of nonfatal myocardial infarction $(p=0.89)$, nonfatal stroke $(p=0.3)$, unstable angina pectoris $(p=0.49)$, and ischemia-driven revascularization ( $p=0.47$ ) with an incline in Cys-C levels. Conclusion: Elevated Cys-C levels were associated with increased all-cause mortality but not cardiovascular events other than mortality in ACS patients.

(c) 2022 The Author(s)

Published by S. Karger AG, Basel

\section{Introduction}

Cystatin- $\mathrm{C}(\mathrm{Cys}-\mathrm{C})$ is a protease inhibitor enzyme produced by almost all human nucleated cells and exists freely in blood without binding to any other protein [1-3]. It is filtered into the renal glomerulus and is almost completely metabolized to amino acids in the proximal tubule. The serum Cys-C level strongly reflects the glomerular filtration rate (GFR) and is considered an indicator of renal

Clinical trial registration No. UMIN000002742, registered as an international standard randomized controlled trial. Registry URL: https:// www.umin.ac.jp.

(C) 2022 The Author(s)

Published by S. Karger AG, Basel

This is an Open Access article licensed under the Creative Commons Attribution-NonCommercial-4.0 International License (CC BY-NC) (http://www.karger.com/Services/OpenAccessLicense), applicable to the online version of the article only. Usage and distribution for commercial purposes requires written permission.
Correspondence to:

Hiroyuki Arashi, arashi.hiroyuki@twmu.ac.jp 
function. Several studies have reported that elevated Cys-C levels were strongly associated with poor clinical outcomes in patients with coronary artery disease [4-6]. Further, studies have shown how Cys-C is associated with mortality in patients with acute coronary syndrome (ACS) [712]. While several studies have analyzed the association between Cys-C and mortality, few studies have compared the relationship between Cys- $\mathrm{C}$ levels and cardiovascular events other than mortality [10]. Moreover, some studies analyzing the relationship between Cys-C levels and cardiovascular events in patients with coronary artery disease have collected data over a couple of decades before [7, 9], but these findings have been inconsistent across studies. In our previous, prospective, randomized controlled study of the Heart Institute of Japan-PRoper level of lipid-lowering with Pitavastatin and Ezetimibe in acute coRonary syndrome (HIJ-PROPER), we compared outcomes of the standard therapy to those of intensive lipid-lowering therapy [13]. The aims of the present subgroup analysis of the HIJ-PROPER study were to examine (1) the distribution of Cys-C levels at baseline, (2) association between Cys-C levels and mortality, and (3) association between Cys-C levels and cardiovascular events other than mortality in ACS patients treated using contemporary measures.

\section{Methods}

\section{Patients}

We conducted a retrospective sub-analysis of data collected in the parent HIJ-PROPER study. Briefly, the HIJ-PROPER study was a multicenter, prospective, randomized controlled trial that compared outcomes of two lipid-lowering treatments and included participants from 19 Japanese hospitals [13]. In total, 1,734 patients diagnosed with ACS were randomized into 2 groups. One group received intensive lipid-lowering therapy (pitavastatin + ezetimibe therapy) and the other group received conventional lipid-lowering therapy (pitavastatin monotherapy) between January 2010 and April 2013. Following the loss of 13 patients to follow-up, the prospective data of 1,721 patients were finally analyzed in the original study. In the present study, we enrolled those patients whose serum Cys-C levels had been determined at baseline, which was defined as within $24 \mathrm{~h}$ of hospitalization with ACS.

\section{Laboratory Investigations}

All the HIJ-PROPER study participants had been hospitalized for the management of ACS, and samples for laboratory testing were collected within $24 \mathrm{~h}$ of admission. Biochemical analyses were exclusively performed at SRL Inc. (Hachioji, Tokyo, Japan), an external laboratory. Values of the estimated GFR (eGFR) used in this study were calculated by applying equations developed by the Japanese Society of Nephrology (male, $194 \times$ serum creatinine $\mathrm{e}^{-1.094} \times$ age $^{-0.287}$ and female, $194 \times$ serum creatinine $\mathrm{e}^{-1.094} \times$ $\left.\operatorname{age}^{-0.287} \times 0.739\right)[14]$.

Elevated Cystatin-C Levels Associated with Increased Mortality in ACS
Analysis and Outcome Measures

The primary endpoint of the current study was all-cause mortality. The secondary endpoint was the same as that in the original HIJ-PROPER study: a composite endpoint of the first occurrence of all-cause mortality, nonfatal myocardial infarction, nonfatal stroke, unstable angina pectoris, or ischemia-driven revascularization. Patients were divided into 4 groups as per quartiles (Q) of Cys-C levels at baseline.

Patient characteristics were compared among these groups. We also examined whether Cys-C levels affected the incidence of the primary and secondary endpoints in patients diagnosed with ACS during a median observation period of 4.0 years.

\section{Statistical Analysis}

Continuous variables were reported as the mean and standard deviation. Nonnormally distributed data are reported as medians and interquartile ranges, while categorical data are presented as absolute values and percentages. Welch's $t$ test and the MannWhitney $U$ test were used to analyze normally and nonnormally distributed data, respectively. Pearson's $\chi^{2}$ test was used for the analysis of categorical data. Among the 4 groups, the time interval until the first occurrence of endpoint events was analyzed using the Kaplan-Meier method along with the log-rank test. The conventional Cox proportional hazard regression model to assess how well quartiles of Cys- $\mathrm{C}$ could predict the mortality and cardiovascular events was used to calculate hazard ratios (HRs) and 95\% confidence intervals (CIs) for each endpoint. Multivariable regression analyses were performed after adjusting for variables including age, sex, comorbidities' prevalence (hypertension and diabetes mellitus), current smoking habit, and revascularization history. Variables included in the multivariable analyses were selected based on the imbalance of the patient characteristics and clinical meaningfulness. Due to the small number of endpoints in the current study, we included selected potential confounders. Multivariable analysis with confounders including Cys-C as a continuous variable was evaluated to identify independent risk factors for the primary endpoint. The area under the curve (AUC) according to receiver operating characteristic curve (ROC) analysis was used to examine the cutoff value of Cys- $\mathrm{C}$ or the eGFR to predict the occurrence of all-cause mortality. A $p$ value of $<0.05$ was considered statistically significant unless stated otherwise. All statistical analyses were performed using the software JMP Pro (Ver. 15; SAS Institute Inc., Cary, NC, USA).

This study was conducted in accordance with the principles of the 1975 Declaration of Helsinki. The Institutional Review Board of each participating medical center or the relevant Ethics Committee approved the protocol, and all patients provided written informed consent for trial enrollment.

\section{Results}

A total of 1,721 patients were enrolled in the original analysis of the HIJ-PROPER study. Cys-C values were unavailable for $621 / 1,721$ patients analyzed in the parent HIJ-PROPER study. Therefore, a total of 1,100 patients were finally included in the current study. Figure 1 shows the flow chart of the current sub-study. Patients excluded 
1. Study flowchart. ACS, acute coro-

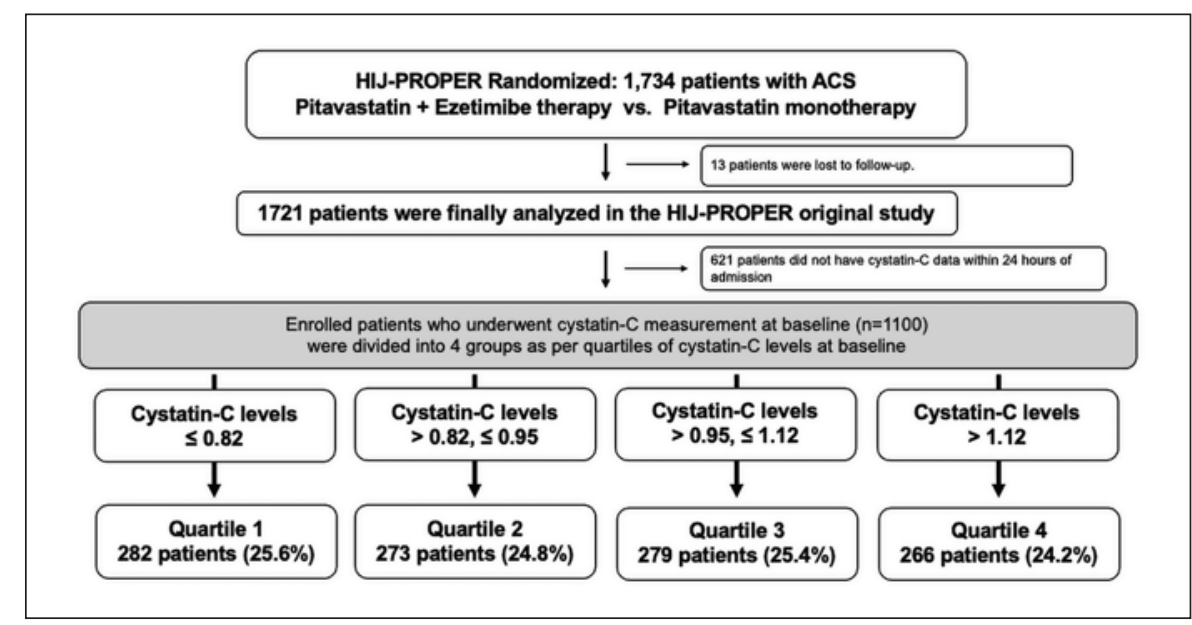
nary syndrome.

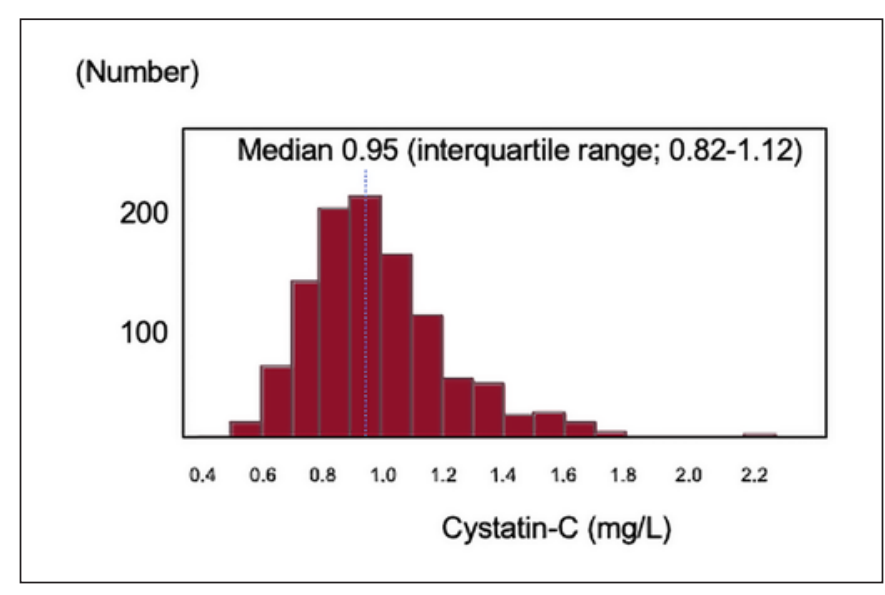

Fig. 2. Distribution of Cys-C $(N=1,100)$.

from the study were comparable to those included in the study in terms of the majority of the baseline characteristics (online suppl. Table. 1; for all online suppl. material, see www.karger.com/doi/10.1159/000522412). However, a different distribution of the clinical classification of ACS was observed between patients included in the study and those excluded from the study. In addition, the prevalence of hypertension was more common in patients excluded from the analysis.

The mean level of Cys-C in this sub-analysis was 0.95 (interquartile range; 0.82-1.12) $\mathrm{mg} / \mathrm{L}$ (Fig. 2). Patients were divided into 4 groups as per quartiles of Cys-C levels at baseline: Q1, $\leq 0.82 \mathrm{mg} / \mathrm{L}$; Q2, from 0.83 to $0.95 \mathrm{mg} / \mathrm{L}$; Q3, from 0.96 to $1.12 \mathrm{mg} / \mathrm{L}$; and Q4 $>1.12 \mathrm{mg} / \mathrm{L}$.

The numbers of patients assigned into Q1-4 were 282 (25.6\%), 273 (24.8\%), 279 (25.4\%), and $266(24.2 \%)$, re- spectively. Baseline clinical characteristics of the 4 groups are summarized in Table 1. Median (interquartile range) of Cys-C levels for patients in Q1-4 were $0.75(0.68,0.79)$, $0.89(0.86,0.93), 1.03(0.99,1.06)$, and $1.3(1.2,1.5) \mathrm{mg} / \mathrm{L}$, respectively. Older patients tended to show higher Cys-C values. Concurrent hypertension and diabetes mellitus or history of revascularization were commonly noted in those with high Cys-C levels. Conversely, such patients were less likely to have a current smoking habit. Patients with elevated levels of Cys-C commonly used renin-angiotensin system inhibitors and calcium channel blockers. Levels of low-density lipoprotein cholesterol and high-density lipoprotein cholesterol were low in patients with elevated Cys-C levels. With regard to angiographical characteristics, patients with elevated levels of Cys-C had high frequency of multivessel lesions.

\section{Incidence of the Primary Endpoint (All-Cause Mortality)}

During a median observation period of 4.0 years (range $0-6.2$ ), the primary endpoint was noted in $5,12,18$, and 36 patients in Q1-Q4, corresponding to an incidence rate of $1.8 \%, 4.4 \%, 6.5 \%$, and $13.5 \%$, respectively $(p<0.0001$ for difference among 4 groups) (Table 2). A Kaplan-Meier curve demonstrated that higher levels of Cys-C were associated with increased all-cause mortality ([HR: 2.74, 95\% CI: $1.02-8.62, p=0.005$, for Q2 vs. Q1] [HR: 4.15, 95\% CI: $1.66-12.6, p=0.002$, for Q3 vs. Q1] [HR: 9.50, 95\% CI: 4.07-27.7, $p<0.0001$, for Q4 vs. Q1]) (Fig. 3). A Cox multivariable proportional hazard regression analysis performed after making necessary adjustments for baseline variables also showed a stepwise increase in HRs for all-cause mortality across all 4 quartiles of Cys-C ( $p=$
Saito/Arashi/Yamaguchi/Mori/Ogawa/ Hagiwara 
Table 1. Baseline clinical characteristics of 4 groups based on Cys-C quartiles ( $\mathrm{mg} / \mathrm{L}$ )

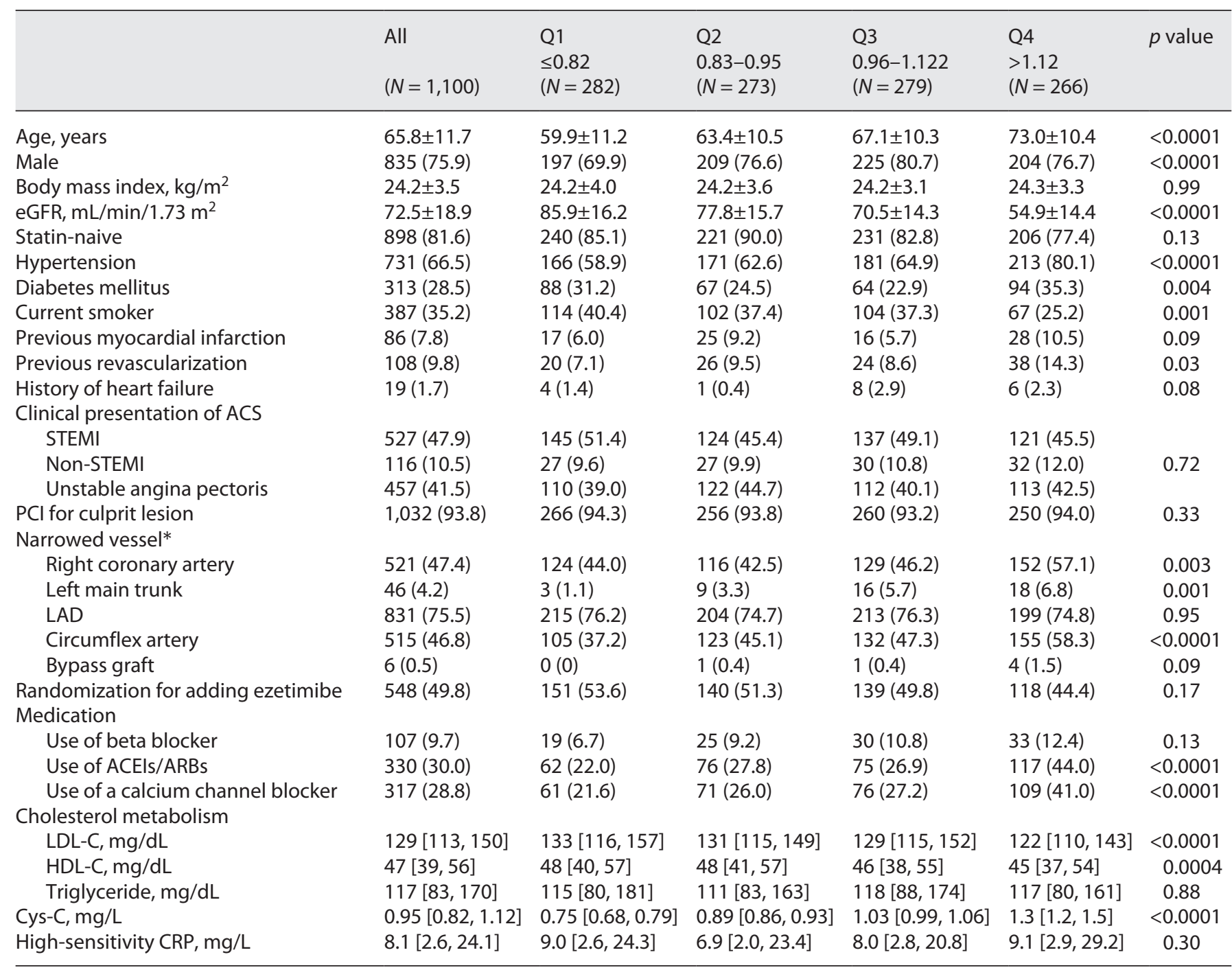

Data are expressed as mean $\pm \mathrm{SD}$, median [interquartile range], or number (percentage). ACEls, angiotensin-converting enzyme inhibitors; ACS, acute coronary syndrome; ARBs, angiotensin II receptor blockers; CRP, C-reactive protein; eGFR, estimated glomerular filtration rate; HDL-C, high-density lipoprotein cholesterol; LDL-C, low-density lipoprotein cholesterol; LAD, left anterior descending artery; $\mathrm{PCl}$, percutaneous coronary intervention; STEMI, ST elevation myocardial infarction; SD, standard deviation. * About 1,100 participants enrolled in the current sub-analysis had a total of 1,919 narrowed vessels, which were considered to indicate significant stenosis with a narrowing of more than $50 \%$.

0.04 , for difference among the 4 groups) (Table 2). A multivariable analysis which was analyzed with Cys- $\mathrm{C}$ as a continuous variable revealed that higher levels of Cys-C and older age were independent predictors of primary endpoints (HR, 2.95, 95\% CI, 1.49-5.33, $p=0.001$ for Cys-C; HR, 1.07, 95\% CI, 1.04-1.10, $p<0.0001$ for age; online suppl. Table 2).

\section{Incidence of the Secondary Endpoint}

The secondary endpoint was noted in $75,91,90$, and 104 patients in Q1-Q4, corresponding to an incidence rate of $26.6 \%, 33.3 \%, 32.3 \%$, and $39.1 \%$, respectively ( $p$ $=0.01$ for difference among 4 groups) (Table 2 ) (online suppl. Fig. 1). A Cox multivariable proportional hazard regression analysis showed no stepwise increase in HRs for secondary endpoint across all 4 quartiles of Cys-C ( $p=0.32$, for difference among the 4 groups). Though, 
Table 2. Distribution of individual components of the primary and secondary endpoints among 4 groups

\begin{tabular}{|c|c|c|c|c|c|}
\hline \multicolumn{6}{|c|}{ Primary endpoint (all-cause mortality) } \\
\hline$N(\%)$ & $5(1.8)$ & $12(4.4)$ & $18(6.5)$ & $36(13.5)$ & \\
\hline \multicolumn{6}{|l|}{$\mathrm{HR}(95 \% \mathrm{Cl})$} \\
\hline \multicolumn{6}{|l|}{ Secondary endpoint* } \\
\hline$N(\%)$ & $75(26.6)$ & $91(33.3)$ & $90(32.3)$ & $104(39.1)$ & \\
\hline \multicolumn{6}{|l|}{$\mathrm{HR}(95 \% \mathrm{Cl})$} \\
\hline Nonadjusted & Reference & $1.37(1.01-1.87)$ & $1.29(0.95-1.76)$ & $1.65(1.23-2.23)$ & 0.01 \\
\hline Adjusted & Reference & $1.29(0.94-1.76)$ & $1.14(0.83-1.57)$ & $1.32(0.94-1.84)$ & 0.30 \\
\hline \multicolumn{6}{|l|}{ Nonfatal MI } \\
\hline \multicolumn{6}{|l|}{$\mathrm{HR}(95 \% \mathrm{Cl})$} \\
\hline \multicolumn{6}{|l|}{ Nonfatal stroke } \\
\hline$N(\%)$ & $3(1.1)$ & $4(1.5)$ & $7(2.5)$ & $7(2.6)$ & \\
\hline \multicolumn{6}{|l|}{$\mathrm{HR}(95 \% \mathrm{Cl})$} \\
\hline Nonadjusted & Reference & $1.51(0.33-7.66)$ & $2.66(0.74-12.4)$ & $3.02(0.83-14.1)$ & 0.30 \\
\hline Adjusted & Reference & $1.30(0.29-5.87)$ & $1.93(0.48-7.82)$ & $1.82(0.41-8.12)$ & 0.78 \\
\hline \multicolumn{6}{|c|}{ Unstable angina pectoris } \\
\hline$N(\%)$ & $9(3.2)$ & $8(2.9)$ & $14(5.0)$ & $8(3.0)$ & \\
\hline \multicolumn{6}{|l|}{$\mathrm{HR}(95 \% \mathrm{Cl})$} \\
\hline Nonadjusted & Reference & $0.97(0.37-2.50)$ & $1.71(0.74-3.96)$ & $1.06(0.41-2.75)$ & 0.49 \\
\hline Adjusted & Reference & $0.87(0.33-2.29)$ & $1.58(0.65-3.80)$ & $0.80(0.28-2.26)$ & 0.39 \\
\hline \multicolumn{6}{|c|}{ Ischemia-driven revascularization } \\
\hline$N(\%)$ & $68(24.1)$ & $74(27.1)$ & $65(23.3)$ & $72(27.1)$ & \\
\hline Adjusted & Reference & $2.29(0.59-8.94)$ & $1.59(0.39-6.53)$ & $4.63(1.27-16.9)$ & 0.03 \\
\hline
\end{tabular}

Adjusted model was adjusted by age, sex, prevalence of hypertension and diabetes mellitus, history of smoking, and revascularization. $\mathrm{Cl}$, confidence interval; HR, hazard ratio. * Secondary endpoint is defined as a composite end point of all-cause death, nonfatal myocardial infarction, nonfatal stroke, unstable angina pectoris, or ischemia-driven revascularization.

a stepwise increase in the incidence rate for all-cause mortality and cardiovascular mortality $(p<0.0001)$ with an incline in Cys-C levels was observed, no difference was observed in the incidence rate for nonfatal myocardial infarction $(p=0.89)$, nonfatal stroke $(p=$ $0.3)$, unstable angina pectoris $(p=0.49)$, and ischemiadriven revascularization $(p=0.47)$ with an incline in Cys-C levels.
All-Cause Mortality according to Age, Sex, and BMI

Online supplementary Table 3 summarizes results of the Cox proportional hazards regression subgroup analysis of all-cause mortality, based on age $(<65$ years or $\geq 65$ years), sex (male/female), and BMI ( $<25 \mathrm{~kg} / \mathrm{m}^{2}$ or $\geq 25 \mathrm{~kg} /$ $\mathrm{m}^{2}$ ) of study participants. A stepwise increase in HR for all-cause mortality was observed among the subgroups regardless of differences in age, sex, and BMI (online suppl. Fig. 2).
Saito/Arashi/Yamaguchi/Mori/Ogawa/ Hagiwara 
Fig. 3. Association between Cys-C quartiles and all-cause mortality. CI, confidence interval; HR, hazard ratio; Q, quartile.

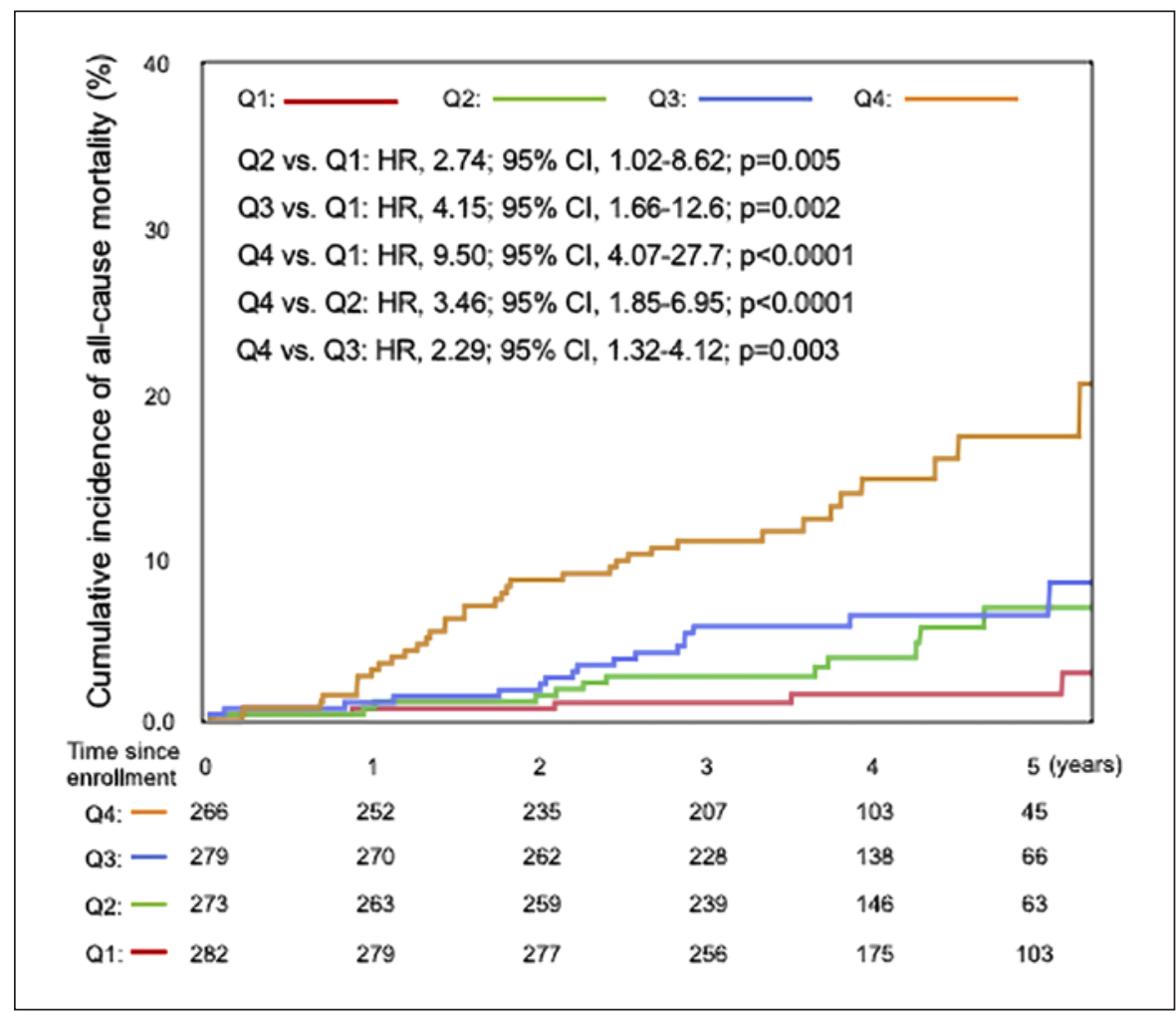

\section{Predictive Status of Cys-C or the eGFR in All-Cause} Mortality

The receiver operating characteristic curve analysis indicated that the cutoff point of Cys-C or the eGFR to predict the occurrence of all-cause mortality were $1.03 \mathrm{mg} / \mathrm{L}$ (Fig. $4 \mathrm{a}$ ) and $65.0 \mathrm{~mL} / \mathrm{min} / 1.73 \mathrm{~m}^{2}$ (Fig. $4 \mathrm{~b}$ ), respectively. The AUC value of Cys- $\mathrm{C}$ to predict all-cause mortality was 0.71 , which was comparable with the AUC value of the eGFR (0.69).

The frequency of all-cause mortality was significantly higher in patients with Cys-C $\geq 1.03 \mathrm{mg} / \mathrm{L}$ than in patients with Cys-C $\leq 1.03$ mg/L (HR: 4.39; 95\% CI: 2.64-7.27; $p<$ 0.0001).

\section{Discussion}

This sub-analysis of the HIJ-PROPER suggests the following: (1) among ACS patients, the median levels of CysC were $0.95 \mathrm{mg} / \mathrm{L}$; (2) Cys-C levels estimated during the early hospitalization period in ACS patients treated using contemporary measures could independently predict allcause mortality; (3) but they cannot predict cardiovascular events other than mortality; and (4) the cutoff value of
Cys-C to predict the occurrence of all-cause mortality was $1.03 \mathrm{mg} / \mathrm{L}$. Several studies have reported the association of Cys-C levels with mortality in patients diagnosed with ACS [7-12]. Most studies suggested that elevated Cys-C levels were independently associated with higher all-cause mortality after adjusting for patients' baseline characteristics and laboratory test results. The SOLID-TIMI 52 subanalysis revealed that the elevated Cys-C levels were not related to increased all-cause mortality in the adjusted model [10]. However, the aforementioned sub-analysis differed from the other studies, with the most notable difference being the timing of Cys-C estimation. In the SOLID-TIMI 52 sub-analysis, blood samples were obtained at a median 14-day time interval after the ACS onset. Conversely, blood samples for Cys-C estimation were obtained at the time of hospital admission during other similar trials, including the present study. The biomarker status of Cys- $\mathrm{C}$ as a predictor of mortality might be affected by the timing of obtaining the blood sample.

Treatment strategy for ACS has changed with the trend moving toward early invasive treatment [15-18]. In the previous studies on Cys- $\mathrm{C}$, the frequency of invasive treatment during hospitalization for ACS varied widely from 16 to $76 \%$. Patients enrolled in the current study received con- 


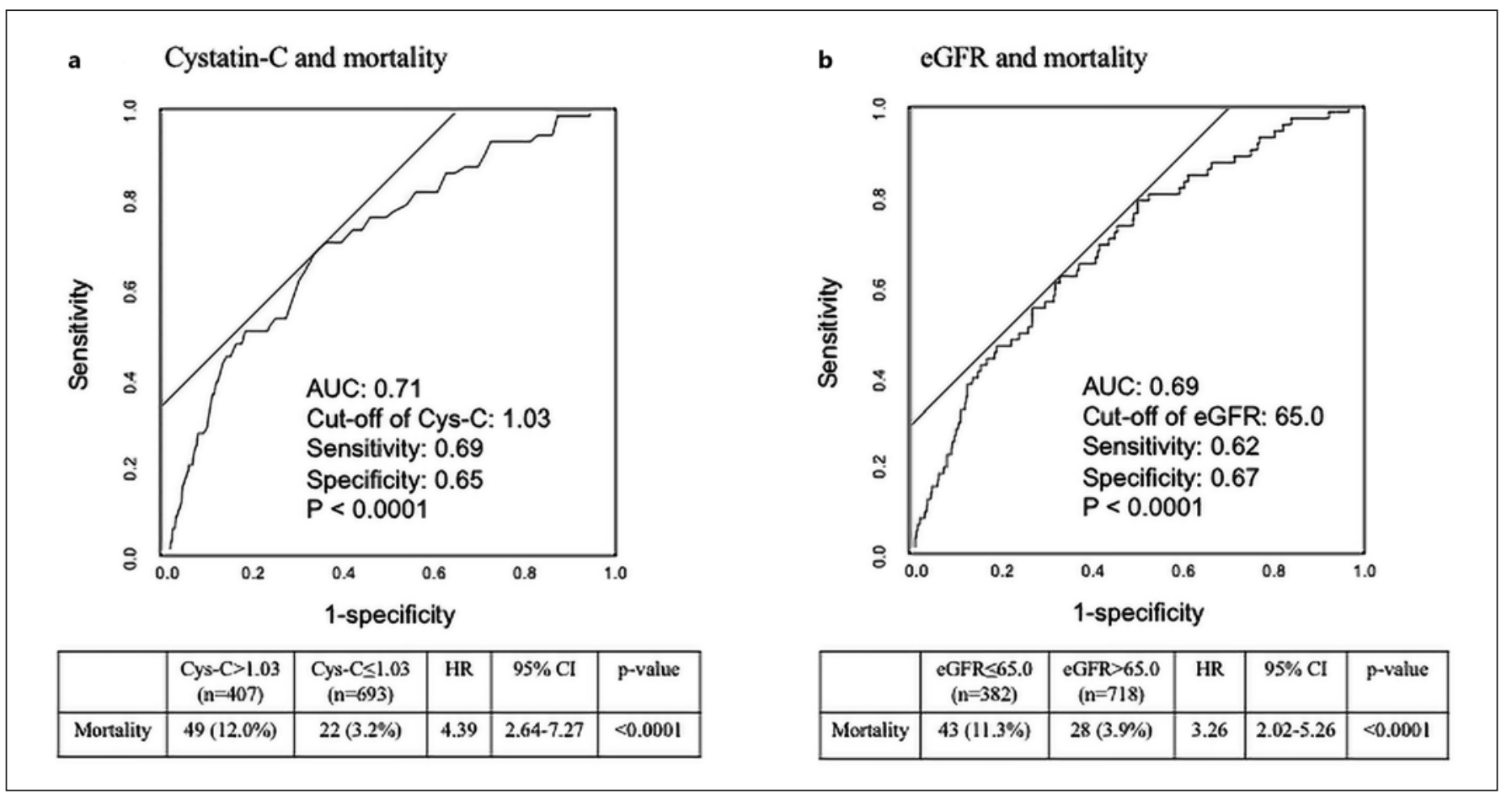

Fig. 4. The AUC according to ROC analysis was used to examine the Cys-C and eGFR cutoff value to predict the occurrence of allcause mortality (Cys-C (a), eGFR (b)). The ROC curve analysis indicated that the Cys- $\mathrm{C}$ and eGFR cutoff points were $1.03 \mathrm{mg} / \mathrm{L}$ and $65.0 \mathrm{~mL} / \mathrm{min} / 1.73 \mathrm{~m}^{2}$, respectively. After dividing patients into 2 groups based on the cutoff point, the incidence rate of allcause mortality was compared between the 2 groups. AUC, area under the curve; Cys-C, cystatin-C; eGFR, estimated glomerular filtration rate; $\mathrm{HR}$, hazard ratio; $\mathrm{CI}$, confidence interval; $\mathrm{ROC}$, receiver operating characteristic curve. temporary ACS treatment. More than $90 \%$ of patients underwent percutaneous coronary intervention during the acute, initial phase of hospitalization in the original HIJPROPER study. Further, enrolled patients had received optimal medical therapy (online suppl. Table 4) and were continuously monitored during the follow-up period. Our result suggested that the Cys-C levels estimated during the early hospitalization period in ACS patients treated using contemporary measures could predict all-cause mortality.

When the analysis was calculated for the relationship between the levels of Cys- $C$ and cardiovascular event other than mortality, the difference in events among the 4 groups disappeared in the current study. The correlation between the levels of Cys- $\mathrm{C}$ and the incidence rate for myocardial infarction and stroke in ACS patients remains controversial [6-10]. Some studies reported a positive correlation in Cys-C levels with an incidence rate for myocardial infarction and stroke in patients after ACS in the nonadjusted model, but no association was observed after the adjusted model $[7,10]$. To the best of our knowledge, there is only 1 study that has demonstrated a posi- tive correlation between Cys- $\mathrm{C}$ levels and incidence of myocardial infarction even with multivariable analysis [9]. Considering the past reports and the current analysis, the correlation in Cys-Clevels with myocardial infarction and stroke is not strong. Few past reports have reported an association between Cys-C levels and the incidence of unstable angina pectoris or ischemia-driven percutaneous coronary intervention in ACS patients. In this respect, the data of this study are meaningful. The Cys-C levels might not predict cardiovascular events other than mortality, including the incidence of myocardial infarction, stroke, unstable angina pectoris, and ischemia-driven percutaneous coronary intervention.

Cys- $\mathrm{C}$ is considered a biomarker of renal function and is not influenced by muscle volume and body mass. In this study, the mortality rate was found to show a stepwise increase with rising Cys-C levels, regardless of differences in age, sex, and BMI. Serum Cys-C levels are known to be less influenced by age and individual muscle mass and are related more strongly with all-cause mortality than serum creatinine values $[19,20]$. Further, its predictive status ap- 
pears to be independent of differences in baseline patient characteristics, including age, sex, and BMI. Risk stratification for predicting all-cause mortality could be performed by estimating the Cys-C level during the early hospitalization period, among ACS patients in contemporary practice.

This study had certain limitations. First, this was a nested, retrospective, subgroup analysis that utilized data from the prospective, parent study. Second, our study population consisted entirely of Japanese patients with ACS, which could affect the generalizability of our findings to non-Japanese participants. Third, only baseline data for Cys-C within $24 \mathrm{~h}$ of hospitalization were available in the current study. We could not assess how did the acute kidney injury, which occurred after admission, affect the value of Cys-C and cardiovascular events. Fourth, there was no information on the change in kidney function or kidney damage in this study. Fifth, the AUC value of Cys-C to predict all-cause mortality was slightly higher than those of the eGFR in the current study. However, it has not been clarified whether which biomarker of renal function is better to predict mortality in ACS patients. In conclusion, elevated Cys-C levels were associated with increased all-cause mortality but not associated with cardiovascular events other than mortality in ACS patients.

\section{Acknowledgments}

We thank the HIJ-PROPER participants as well as the investigators and administrative staff of the original parent study for their contributions. The clinical centers that participated in this study were the Tokyo Women's Medical University, the Sakakibara Heart Institute, the Saisei-Kai Kumamoto Hospital, the Cardiovascular Center of Sendai, Seirei Hamamatsu General Hospital, the Saisei-Kai Kurihashi Hospital, the National Yokohama Medical Center, the Tokyo Metropolitan Tama Medical Center, Kosei, General Hospital, NTT (Nippon Telegraph and Telephone corporation)-East Kanto Medical Hospital, Tokyo, Metropolitan Tama-Hokubu Medical Center, Shin-MatsudoCentral General Hospital, JCHO (Japan Community Health care Organization) Sagamino Hospital, Nishiarai Heart Center, Ogikubo Hospital, Shiseikai-Daini Hospital, Tokyo Metropolitan Ebara Hospital, Tokyo Women's Medical University Medical Center East, and Tokyo Women's Medical University Yachiyo Medical Center. We also thank Editage (www.editage.com) for English language editing and publication support.

\section{Statement of Ethics}

Patients or the public were not involved in the design or conduct or reporting or dissemination plans of our research. The study protocol conforms to the ethical guidelines laid within the 1975 Declaration of Helsinki, as reflected in a priori approval provided by the institutional review board or the relevant Ethics Com- mittee of each participating medical center. Before enrollment in the parent trial, written informed consent was obtained from all patients, parents, and/or legal guardians if the participants were under 18 years old. This sub-analysis is nested within the original HIJ-PROPER study, which is registered in the UMIN Registry as an international standard randomized controlled trial (trial No. UMIN000002742, registry URL: https://www.umin.ac.jp).

\section{Conflict of Interest Statement}

The authors have the following disclosures: All members of the HIJ-PROPER study group have received research support to perform clinical trials, from the Japan Research Promotion Society for Cardiovascular Diseases, which is sponsored by the Abbott Vascular Japan Co., Ltd.; AstraZeneca K.K.; Bayer Yakuhin Ltd.; Boston Scientific Corporation; Bristol-Myers K.K.; Daiichi Sankyo Co., Ltd.; Kowa Pharmaceutical Co., Ltd.; Mochida Pharmaceutical Co., Ltd.; MSD K.K.; Nippon Boehringer Ingelheim Co., Ltd.; Novartis Pharma K.K.; Pfizer Japan Inc.; Sanofi K.K.; and Takeda Pharmaceutical Co., Ltd. N.H. reports receiving honoraria from Bristol-Myers K.K. and Nippon Boehringer Ingelheim Co., Ltd., and grants from Astellas Pharma Inc.; Daiichi Sankyo Co., Ltd.; Eisai Co., Ltd.; Mitsubishi Tanabe Pharma Corporation; Otsuka Pharmaceutical Co., Ltd.; Shionogi \& Co., Ltd.; and Takeda Pharmaceutical Co., Ltd. J.Y. belongs to the Clinical Research Division for Cardiovascular Catheter Intervention, which is financially maintained by donations from Abbott Vascular, Boston Scientific, Medtronic, and Terumo. The HIJ-PROPER Steering Committee has full access to all data of the present study and were responsible for the decision to submit the study for publication.

\section{Funding Sources}

The original trial was funded by the Japan Research Promotion Society for Cardiovascular Diseases. No additional extramural funding was used to support this work.

\section{Author Contributions}

H.A., J.Y., H.O., and N.H. conceptualized and designed the original study. H.A. collected data and enrolled and followed up with patients. T.S. and H.A. analyzed and interpreted the collected data. T.S. and H.A. drafted and wrote the manuscript. F.M., J.Y., H.O., and N.H. reviewed the manuscript. All the authors, external and internal, had full access to all data (including statistical reports and tables) in the study and take responsibility for the integrity of the data and accuracy of the analysis.

\section{Data Availability Statement}

All data generated or analyzed during this study are included in this article and its online supplementary material Files. Further inquiries can be directed to the corresponding author. 


\section{References}

1 Inker LA, Schmid CH, Tighiouart H, Eckfeldt JH, Feldman HI, Greene T, et al. Estimating glomerular filtration rate from serum creatinine and cystatin C. N Engl J Med. 2012;367: 20-9.

2 Coll E, Botey A, Alvarez L, Poch E, Quinto L, Saurina A, et al. Serum cystatin $C$ as a new marker for noninvasive estimation of glomerular filtration rate and as a marker for early renal impairment. Am J Kidney Dis. 2000;36: 29-34.

3 Stevens LA, Schmid CH, Greene T, Li L, Beck GJ, Joffe MM, et al. Factors other than glomerular filtration rate affect serum cystatin C levels. Kidney Int. 2009;75:652-60.

4 Shlipak MG, Sarnak MJ, Katz R, Fried LF, Seliger SL, Newman AB, et al. Cystatin C and the risk of death and cardiovascular events among elderly persons. N Engl J Med. 2005; 352:2049-60.

$5 \mathrm{Wu}$ CK, Lin JW, Caffrey JL, Chang MH, Hwang JJ, Lin YS. Cystatin C and long-term mortality among subjects with normal creatinine-based estimated glomerular filtration rates: NHANES III (Third National Health and Nutrition Examination Survey). J Am Coll Cardiol. 2010;56:1930-6.

6 Ix JH, Shlipak MG, Chertow GM, Whooley MA. Association of cystatin C with mortality, cardiovascular events, and incident heart failure among persons with coronary heart disease: data from the Heart and Soul Study. Circulation. 2007;115:173-9.

7 Jernberg T, Lindahl B, James S, Larsson A, Hansson LO, Wallentin L. Cystatin C: a novel predictor of outcome in suspected or confirmed non-ST-elevation acute coronary syndrome. Circulation. 2004;110:2342-8.

8 Taglieri N, Fernandez-Berges DJ, Koenig W, Consuegra-Sanchez L, Fernandez JM, Robles NR, et al. Plasma cystatin C for predic- tion of 1-year cardiac events in Mediterranean patients with non-ST elevation acute coronary syndrome. Atherosclerosis. 2010; 209:300-5.

9 Windhausen F, Hirsch A, Fischer J, van der Zee PM, Sanders GT, van Straalen JP, et al. Cystatin C for enhancement of risk stratification in non-ST elevation acute coronary syndrome patients with an increased troponin T. Clin Chem. 2009;55:1118-25.

10 Correa S, Morrow DA, Braunwald E, Davies RY, Goodrich EL, Murphy SA, et al. Cystatin $\mathrm{C}$ for risk stratification in patients after an acute coronary syndrome. J Am Heart Assoc. 2018;7:e009077.

11 Ristiniemi N, Lund J, Tertti R, Christensson A, Ilva T, Porela $\mathrm{P}$, et al. Cystatin $\mathrm{C}$ as a predictor of all-cause mortality and myocardial infarction in patients with non-ST-elevation acute coronary syndrome. Clin Biochem. 2012;45:535-40.

12 Akerblom Å, Wallentin L, Siegbahn A, Becker RC, Budaj A, Buck K, et al. Cystatin C and estimated glomerular filtration rate as predictors for adverse outcome in patients with STelevation and non-ST-elevation acute coronary syndromes: results from the Platelet Inhibition and Patient Outcomes study. Clin Chem. 2012;58:190-9.

13 Hagiwara N, Kawada-Watanabe E, Koyanagi R, Arashi H, Yamaguchi J, Nakao K, et al. Low-density lipoprotein cholesterol targeting with pitavastatin + ezetimibe for patients with acute coronary syndrome and dyslipidaemia: the HIJ-PROPER study, a prospective, openlabel, randomized trial. Eur Heart J. 2017;38: 2264-76.

14 Matsuo S, Imai E, Horio M, Yasuda Y, Tomita K, Nitta K, et al. Collaborators developing the Japanese equation for estimated GFR. Revised equations for estimated GFR from se- rum creatinine in Japan. Am J Kidney Dis. 2009;53:982-92.

15 O'Donoghue M, Boden WE, Braunwald E, Cannon CP, Clayton TC, de Winter RJ, et al. Early invasive vs conservative treatment strategies in women and men with unstable angina and nonST-segment elevation myocardial infarction: a meta-analysis. JAMA. 2008;300:71-80.

16 Lemesle G, Laine M, Pankert M, Boueri Z, Motreff P, Paganelli F, et al. Optimal timing of intervention in NSTE-ACS without pretreatment: the EARLY randomized trial. JACC Cardiovasc Interv. 2020;13:907-17.

17 Amsterdam EA, Wenger NK, Brindis RG, Casey DE Jr, Ganiats TG, Holmes DR Jr, et al. 2014 AHA/ACC guideline for the management of patients with non-ST-elevation acute coronary syndromes: a report of the American College of Cardiology/American Heart Association Task Force on practice guidelines. J Am Coll Cardiol. 2014;64:139-228.

18 Roffi M, Patrono C, Collet JP, Mueller C, Valgimigli M, Andreotti F, et al. 2015 ESC guidelines for the management of acute coronary syndromes in patients presenting without persistent ST-segment elevation: task force for the management of acute coronary syndromes in patients presenting without persistent st segment elevation of the European Society of Cardiology (ESC). Eur Heart J. 2016; 37:267-315.

19 Shlipak MG, Wassel Fyr CL, Chertow GM, Harris TB, Kritchevsky SB, Tylavsky FA, et al. Cystatin $\mathrm{C}$ and mortality risk in the elderly: the health, aging, and body composition study. J Am Soc Nephrol. 2006;17:254-61.

20 Astor BC, Levey AS, Stevens LA, Van Lente F, Selvin E, Coresh J. Method of glomerular filtration rate estimation affects prediction of mortality risk. J Am Soc Nephrol. 2009;20: 2214-22. 